\title{
Direct and Alternate Current Conductivity and Magnetoconductivity of Nanocrystalline Cadmium-Zinc Ferrite below Room Temperature
}

\author{
Somenath Ghatak ${ }^{1}$, Goutam Chakraborty ${ }^{1}$, Monika Sinha ${ }^{2}$, Swapan Kumar Pradhan ${ }^{2}$, \\ Ajit Kumar Meikap ${ }^{1^{*}}$ \\ ${ }^{1}$ Department of Physics, National Institute of Technology, Deemed University, Durgapur, India; ${ }^{2}$ Department of Physics, University \\ of Burdwan, Burdwan, India \\ E-mail: meikapnitd@yahoo.com
}

Received December 15 $5^{\text {th }}, 2010$; revised January $4^{\text {th }}, 2011$; accepted March $4^{\text {th }}, 2011$.

\begin{abstract}
Nanocrystalline cadmium-zinc ferrite samples were prepared by ball milling method and its electrical transport property were investigated within a temperature range $77 \mathrm{~K} \leq T \leq 300 \mathrm{~K}$ in presence of a magnetic field up to $1 T$ and in a frequency range $20 \mathrm{~Hz}$ to $1 \mathrm{MHz}$. The investigated samples follow a simple hopping type charge transport. The dc magnetoconductivity has been explained in terms of orbital magnetoconductivity theory. The alternating current conductivity follows the universal dielectric response $\sigma(f) \propto T^{n} f^{\prime}$. The values of 's' have a decreasing trend with temperature. The temperature exponent ' $n$ ' depends on frequency. The dielectric permittivity of the samples depends on the grain resistance and interfacial grain boundary resistance. The ac magnetoconductivity is positive which can be explained in terms of impedance of the sample.
\end{abstract}

Keywords: Nanostructures, X-Ray Diffraction, Electrical Properties, Dielectric Properties

\section{Introduction}

The potential application and unusual properties of nanocrystalline materials has made them an object of interest for many researches. The unique properties of these classes of materials come due to the presence of its atoms at the grain boundaries or interfacial boundaries in comparison to coarse grained polycrystalline counterparts. Nanocrystalline spinel ferrite is a group of technologically important nanomaterials that has a potential application in magnetic, electronic and microwave fields [1-5]. Due to their relatively insulating behaviour, they are used as high frequency magnetic materials [6]. In various fields like magnetic recording medium, information storage, colour imaging, bio-processing magnetic refrigeration and magneto optical devices [7-9], the use of nanoferrites has made them a very important material for industrial application where as the reduction of particle size to nanometre scale level, different new mechanism like super paramagnetism, quantum mechanical tunnelling, spin canting etc. makes them interesting among scientists to study their transport properties
$[10,11]$.

The general formula of ferrite are represented as $\mathrm{M}^{2+} \mathrm{Fe}_{2}^{3+} \mathrm{O}_{4}^{2-}$, where $\mathrm{M}$ is a divalent metallic ion. The spinel structure has a unit cell that consists of a cubic close-packed array of 32 oxygen ions with 64 tetrahedral sites ( $\mathrm{T}$ sites) and 32 octahedral sites (O sites); but only eight of the $\mathrm{T}$ sites and 16 of the $\mathrm{O}$ sites are filled. A large number of investigations on structural and magnetic properties like magnetisation measurement, Mossbauer spectroscopy, neutron scattering etc. have been done on the spinel oxide nanoparticles over the last few years [10-23]. The dielectric behaviour on high energy ball milling ultrafine Zinc ferrite above room temperature was reported by Shenoy et al. [17]. They suggested that the defects caused by the milling, produce traps in the surface layer contributes to dielectric permittivity via spin polarised electron tunnelling between grains. Ravinder et al. [24-26] studied the electrical conductivity of cadmium substituted manganese ferrite and cadmium substituted copper ferrites and nickel ferrites above room temperatures. The electrical conduction in these ferrites was explained on the basis of the hopping mechanism. 
They show a transition near the Curie temperature in the conductivity versus temperature curve and also suggested the activation energy in the ferromagnetic region is in general less than that in the paramagnetic region. But a systematic analysis on the electron transport mechanism of cadmium substituted zinc ferrite below room temperature is still lacking.

Thus, the detailed electrical transport properties like ac and dc conductivity, ac and dc magnetoconductivity and dielectric properties of nanocrystalline $\mathrm{Cd}-\mathrm{Zn}$ ferities reported within a temperature range 77 to $300 \mathrm{~K}$ and a frequency range $20 \mathrm{~Hz}$ to $1 \mathrm{MHz}$ in presence as well as absence of a magnetic field up to $1 \mathrm{~T}$.

\section{Experimental}

Accurately weighed starting powders of $\mathrm{CdO}(\mathrm{M} / \mathrm{S}$ Merck, $98 \%$ purity), $\mathrm{ZnO}$ (M/S Merck, 99\% purity) and $\alpha-\mathrm{Fe}_{2} \mathrm{O}_{3}$ (M/S Glaxo, 99\% purity) taken in 0.5:0.5:1 mol\% were hand-ground by an agate mortar pestle in a doubly distilled acetone medium for more than $5 \mathrm{~h}$. The dried homogeneous powder mixture was then termed as unmilled $(0 \mathrm{~h})$ stoichiometric homogeneous powder mixture. A part of this mixture was ball milled for $3 \mathrm{~h}, 8 \mathrm{~h}$, $20 \mathrm{~h}$ and $25 \mathrm{~h}$ duration at room temperature in air in a planetary ball mill (Model P5, M/S Fritsch, GmbH, Germany), keeping the disk rotation speed $=300 \mathrm{rpm}$ and that of the vials $\sim 50 \mathrm{rpm}$ respectively. Milling was done in hardened chrome steel vial of volume $80 \mathrm{ml}$ using 30 hardened chrome steel ball of $10 \mathrm{~mm}$ dia, at ball to powder mass ratio $40: 1$.

The X-ray powder diffraction profiles of unmilled and all ball milled samples were recorded (step size $=0.02^{\circ}$ $\Delta(2 \theta)$, counting time $=5 \mathrm{~s}$, angular range $=15^{\circ}-80^{\circ} 2 \theta$ ) using Ni-filtered $\mathrm{CuK}_{\alpha}$ radiation from a highly stabilized and automated Philips generator (PW1830) operated at $40 \mathrm{kV}$ and $20 \mathrm{~mA}$. The generator is coupled with a Philips X-ray powder diffractometer consisting of a PW 3710 mpd controller, PW1050/37 goniometer and a proportional counter.

The Rietveld's analysis based on structure and microstructure refinement method [27-31] has been employed which is considered to be the best method for microstructure characterization and quantitative estimations of multiphase nanocrystalline material containing several number of overlapping reflections of ferrite phase for both the unmilled and ball milled powder sample.

The electrical conductivity of the samples was measured by a standard four probe method by using $8^{1} / 2$-digit Agilent 3458 multimeter and 6514 Keithley Electrometer. The ac measurement was carried out with a 4284A "Agilent Impedance" analyzer up to the frequency 1 $\mathrm{MHz}$ at different temperatures. Liquid nitrogen cryostat was used to study the temperature dependent conductivity by the ITC 502S Oxford temperature controller. To measure the ac response, samples were prepared as $1 \mathrm{~cm}$ dia pellets by pressing the powder under a hydraulic pressure of $500 \mathrm{MPa}$. Fine copper wires were used as the connecting wire and silver paint was used as coating materials. The experimental density of the pressed pellets has been calculated from the relation $\rho_{\text {exp }}=m / \pi r^{2} h$, where, $m, r$ and $h$ are mass, radius and thickness of the pellet respectively. The measured density lies in the range $5.12 \mathrm{~g} / \mathrm{cc}$ to $6.38 \mathrm{~g} / \mathrm{cc}$. The percentage of error in determining the density is very small $(0.21 \%)$. On the other hand density has been calculated from X-ray data by using the relation $\rho_{\text {theo }}=8 M / N_{A} V_{\text {cell }}$, where, $\mathrm{M}$ is the molar mass of the sample, $\mathrm{N}_{\mathrm{A}}$ is the Avogadro's number and $V_{\text {cell }}$ is the unit cell volume. It is observed that the $d_{e x p}$ is $80 \%$ to $84 \%$ of $d_{\text {theo }}$ for the investigated samples. The capacitance $\left(C_{P}\right)$ and the dissipation factor $(D)$ were measured at various frequencies and temperatures. The real part of ac conductivity, real and imaginary part of dielectric permittivity have been calculated using the relations $\sigma^{\prime}(f)=2 \pi f \varepsilon_{0} \varepsilon^{\prime \prime}(f), \varepsilon^{\prime}(f)=\mathrm{C}_{\mathrm{P}} \mathrm{d} / \varepsilon_{0} \mathrm{~A}$ and $\varepsilon^{\prime \prime}(f)=$ $\varepsilon^{\prime}(f) D$ respectively, where $\varepsilon_{0}=8.854 \times 10^{-12} \mathrm{~F} / \mathrm{m}, \mathrm{A}$ and $\mathrm{d}$ are the area and thickness of the sample respectively. $C_{P}$ is the capacitance measured in Farad; $\mathrm{f}$ is the frequency in $\mathrm{Hz}$. The magnetoconductivity was measured in the same manner varying the transverse magnetic field $\mathrm{B}$ $\leq 1 \mathrm{~T}$ by using an electromagnet.

\section{Results and Discussion}

The XRD powder patterns recorded from unmilled and ball milled powder mixture of $\mathrm{CdO}, \mathrm{ZnO}$ and $\alpha-\mathrm{Fe}_{2} \mathrm{O}_{3}$ are shown in Figure 1. The powder pattern of unmilled mixture contains only the individual reflections of $\mathrm{ZnO}$, $\mathrm{CdO}$ and $\alpha-\mathrm{Fe}_{2} \mathrm{O}_{3}$ phases. The intensity ratios of individual reflections are in accordance with the stoichiometric composition of the mixture. It is evident from the figure that the particle size of the starting materials reduces very fast as their peaks become broadened rapidly in the course of milling. The ferrite reflections were appeared clearly in $3 \mathrm{~h}$ milled sample and the content of ferrite phase increases continuously with milling time as noticed up to $25 \mathrm{~h}$ of milling. The $\mathrm{CdO}$ phase was not used up completely in the process even after $25 \mathrm{~h}$ of milling, whereas the other two starting phases vanishes completely in the course of milling. This indicates to the fact that the formed ferrite phase is a non-stoichiometric in composition. There must be a number of vacancies in the tetrahedral sites of the spinel ferrite lattice due to these unreacted $\mathrm{Cd}^{2+}$ ions.

Figure 2 shows the Rietveld's fitting outputs of unmilled and all ball milled powder patterns. Peak positions 


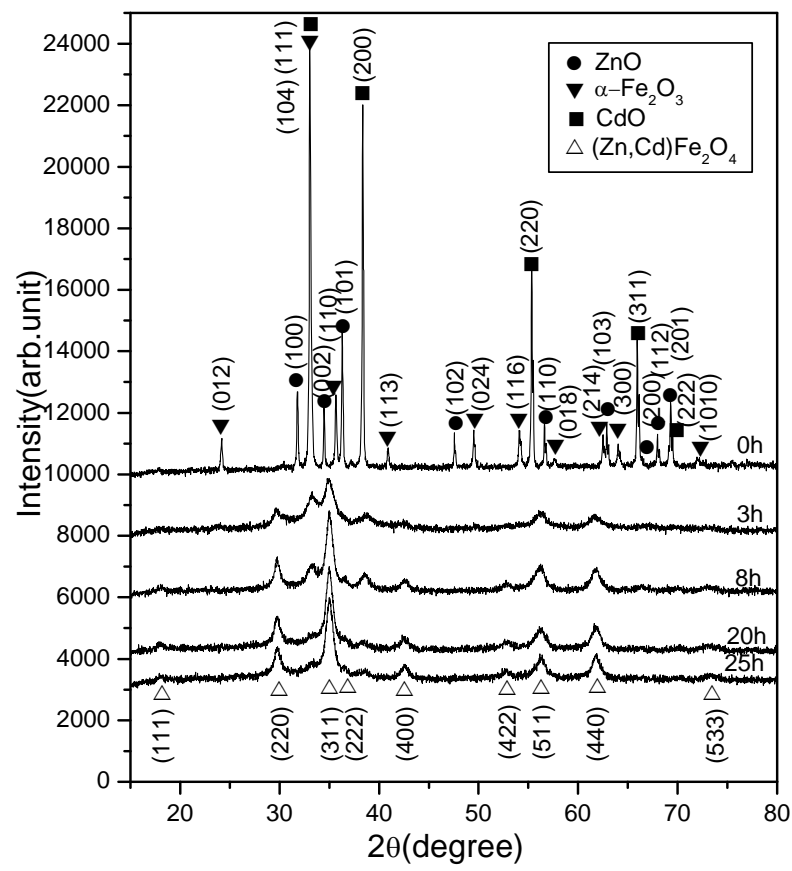

Figure 1. X-ray diffraction patterns of unmilled $(0 \mathrm{~h})$ and ball-milled $\mathrm{CdO}+\mathrm{ZnO}+\alpha-\mathrm{Fe}_{2} \mathrm{O}_{3}$ powder mixture for different durations of ball-milling.

of all reflections of all four phases are marked and shown at the bottom of the plot. Residual of fitting $\left(I_{0}-I_{c}\right)$ between observed $\left(I_{0}\right)$ and calculated $\left(I_{c}\right)$ intensities of each fitting is plotted under respective patterns. In the present analysis, all recorded XRD patterns of ball milled samples were fitted well only with normal spinel ferrite

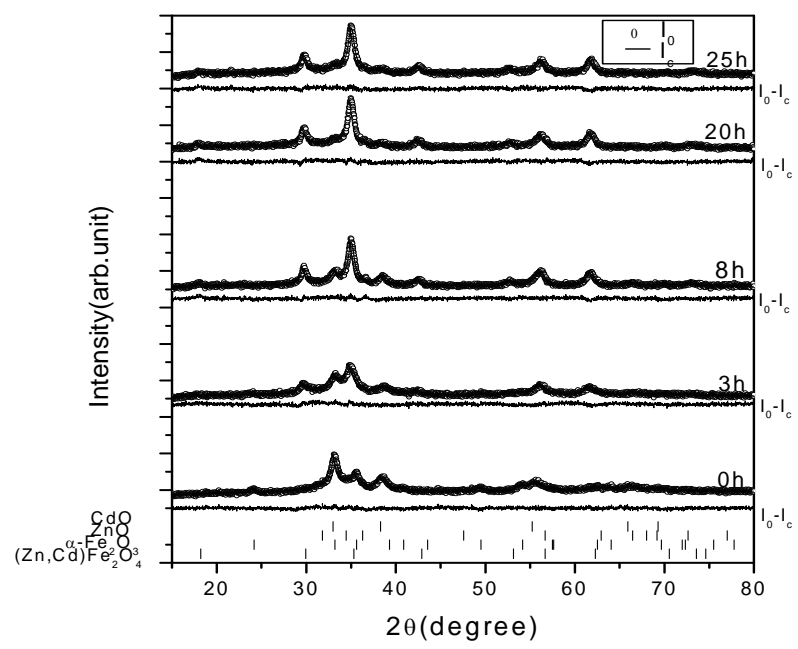

Figure 2. Observed (o) and calculated (-) XRD patterns of unmilled $(0 \mathrm{~h})$ and different ball-milled samples revealed from Rietveld's powder structure refinement analysis. Residues of fittings are shown under the respective patters. Peak positions of the phases are shown at the base line. phase. It indicates that there are tetrahedral vacancies in normal spinel structure of $(\mathrm{Zn}, \mathrm{Cd})$ ferrite which are supposed to fill with $\mathrm{Cd}^{2+}$ ions.

Figure 3 shows the variation of relative phase abundances of different phases with increasing milling time. The content (mole fraction) of $\mathrm{ZnO}$ phase decreases very rapidly and after $3 \mathrm{~h}$ of milling it becomes almost nil, whereas the variation of $\mathrm{CdO}$ content shows that initially the phase was utilized rapidly in ferrite phase but at the higher milling time, the rate of inclusion becomes very slow and till $25 \mathrm{~h}$ milling the phase was not completely incorporated in the ferrite matrix and $\sim 0.04$ mole fraction of the phase remained unreacted. The $\alpha-\mathrm{Fe}_{2} \mathrm{O}_{3}$ phase content decreases in a moderate rate and after $8 \mathrm{~h}$ of milling it almost vanishes. The ferrite phase content increases sharply up to $8 \mathrm{~h}$ of milling and then approaches towards a saturation to $\sim 0.96$ mole fraction (Table 1). It may be noticed that the ferrite content increases considerably until the $\alpha-\mathrm{Fe}_{2} \mathrm{O}_{3}$ phase was used up completely and after that a slight increment up to $25 \mathrm{~h}$ milling is due to a very slow diffusion of $\mathrm{Cd}^{2+}$ ions into the ferrite matrix. All these variations in contents indicate that $\mathrm{Zn}^{2+}$ ions have occupied the tetrahedral positions quite rapidly but the $\mathrm{Cd}^{2+}$ ions took longer time and even after $25 \mathrm{~h}$ of milling some tetrahedral positions remained vacant due to insolubility of $\mathrm{CdO}$ in ferrite matrix. It is therefore obvious that the prepared ferrite phase is a Zn-rich non-stoichiometric $(\mathrm{Zn}, \mathrm{Cd}) \mathrm{Fe}_{2} \mathrm{O}_{4}$ normal spinel with tetrahedral vacancies.

The nature of variation of lattice parameter of the cubic $(\mathrm{Zn}, \mathrm{Cd}) \mathrm{Fe}_{2} \mathrm{O}_{4}$ phase with increasing milling time is shown in Figure 4. It can be seen from the plot that the lattice parameter of ferrite phase formed after $1 \mathrm{~h}$ of milling reduces rapidly within $3 \mathrm{~h}$ of milling from the value $0.859 \mathrm{~nm}$ to $\sim 0.849 \mathrm{~nm}$ (Table 1) and then remained almost invariant up to $25 \mathrm{~h}$ of milling. In the course of milling, $\mathrm{ZnO}$ phase utilized completely in tetrahedral vacancies but $\mathrm{CdO}$ phase was not utilized completely and some tetrahedral sites remained unoccupied even after 25 $\mathrm{h}$ of milling.

The scanning electron micrograph of the samples CZF3h and CZF20h are shown in Figures 5(a) and 5(b). The pictures show that the samples are not closely packed and consist of several grains. The grains are well resolved and have almost circular (spherical) shape. It is clearly seen in the micrographs that the grains are at nanoscale. The average grain size determined from SEM was noted as $35 \mathrm{~nm}-40 \mathrm{~nm}$.

The dc conductivity of the Cd-Zn ferrite samples have been measured in the temperature range $77 \mathrm{~K} \leq T \leq 300$ $\mathrm{K}$. It is observed that the incorporation of Cadmium atom within zinc ferrite reduces the dc conductivity in compare to our previous study [32]. The room temperature con- 


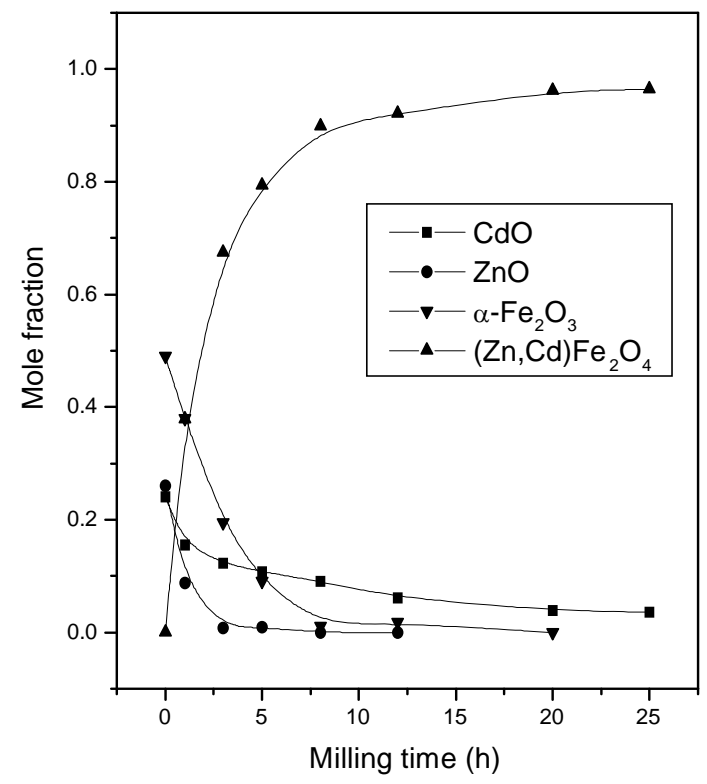

Figure 3. Variations of mole fraction of different phases in ball-milled $\mathrm{CdO}+\mathrm{ZnO}+\alpha-\mathrm{Fe}_{2} \mathrm{O}_{3}$ powder mixture with increasing milling time.

ductivity $\sigma(300 \mathrm{~K})$ and conductivity ratio $\sigma_{\mathrm{r}}[=\sigma(300 \mathrm{~K}) /$ $\sigma(77 \mathrm{~K})]$ of the investigated samples increases with the increasing of milling time. The value of conductivity ratio changes between $1.02 \times 10^{2}$ to $2.48 \times 10^{3}$ which is smaller than $\mathrm{Zn}$-ferrite $\left(\sigma_{\mathrm{r}}=1.12 \times 10^{3}\right.$ to $\left.1.21 \times 10^{6}\right)$ [32]. The contacts between the nanoparticles are the reason behind this larger conductivity ratio. The conductivity variation of all the samples has the characteristic of a semiconductor i.e. their conductivity increases with in-

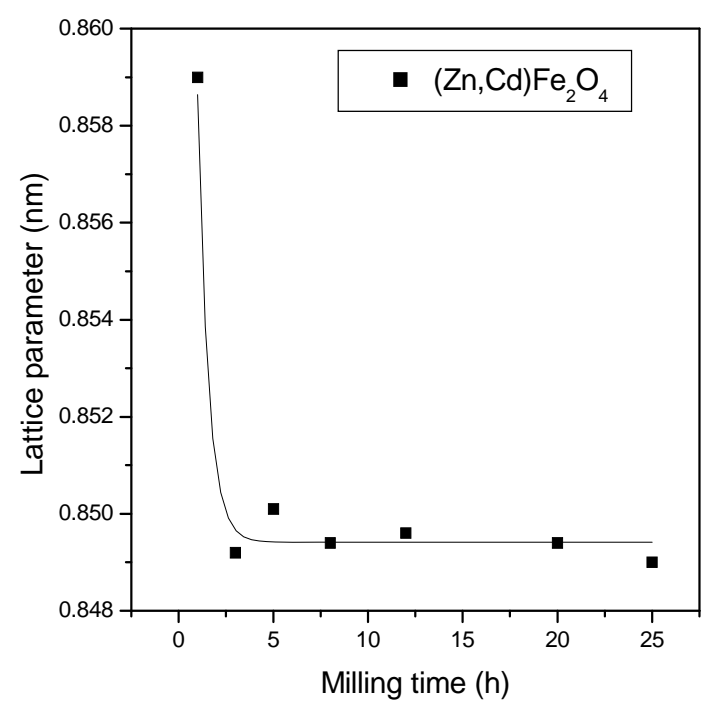

Figure 4. Variation of lattice parameter of the (Zn,Cd)$\mathrm{Fe}_{2} \mathrm{O}_{4}$ phase with increasing milling time.

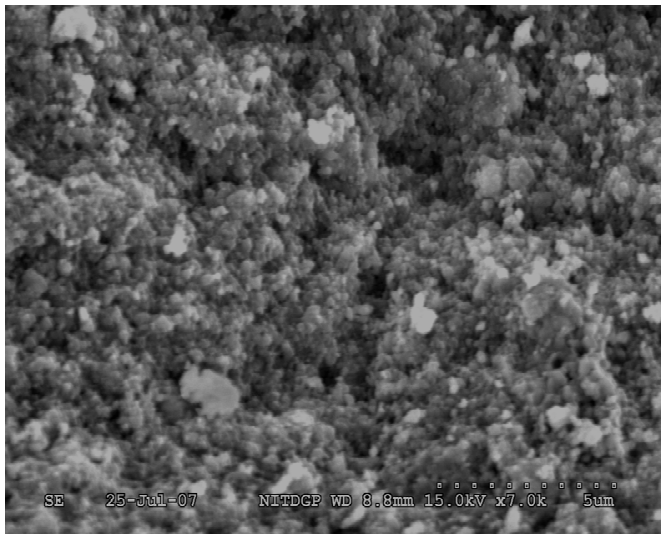

(a)

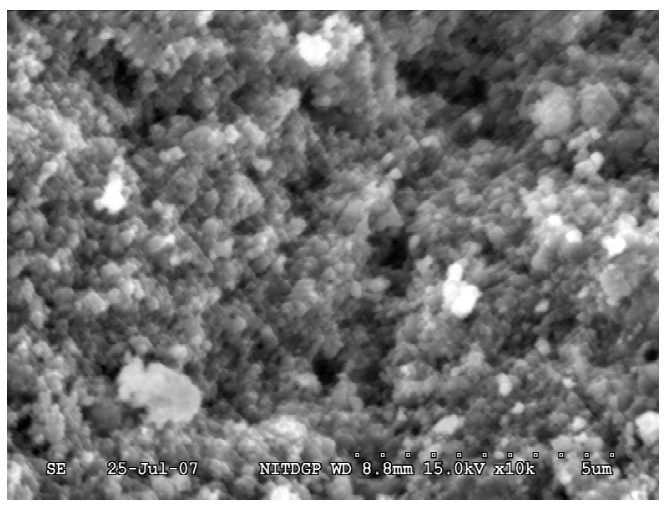

(b)

Figure 5. Scanning electron micrograph of (a) CZF3h and (b) CZF20h.

creasing temperature. The variation of conductivity with temperature is indicated in Figure 6. Lu et al. [18] reported the similar type of variation of conductivity in case of nanocrystalline Zinc ferrite samples. Figure 6 shows the linear variation of $\ln [\sigma(T)]$ with $1 / T$ indicating a simple hopping type charge transport in all the investigated samples [33]. The values of activation energy of different samples have been obtained from the slopes of different straight lines. The values of $E_{a}$ with milling time are shown in the inset of Figure 6. The values of $E_{a}$ increase with increasing milling time and hence with decreasing particle size of the samples. The increasing milling time may decrease the size of the metal core which in turn increases the activation energy.

The influence of magnetic field on the dc conductivity of the samples has been observed under a magnetic field of strength $<1 \mathrm{~T}$. The magnetoconductivity ratio of the milled samples increases with increasing milling time which may be explained in terms of a simple phenomenological model named as orbital magnetoconductivity theory (Forward interference model) [34,35]. Again, for the unmilled sample, there is a decrease in conductivity 
Table 1. Microstructure parameters of unmilled and ball-milled $\mathrm{CdO}$, $\mathrm{ZnO}$ and $\alpha$-Fe $\mathrm{O}_{2} \mathrm{O}_{3}$ powder mixture as revealed from Rietveld's analysis of XRD data.

\begin{tabular}{|c|c|c|c|c|c|}
\hline $\begin{array}{l}\text { Milling } \\
\text { Time }\end{array}$ & Phase present & $\begin{array}{c}\text { Mole } \\
\text { fraction } \\
\pm\left(10^{-3}-10^{-2}\right)^{*}\end{array}$ & $\begin{array}{l}\text { Lattice } \\
\mathrm{a}(\mathrm{nm})\end{array}$ & $\begin{array}{l}\left.10^{-3}\right)^{*} \\
\mathrm{c}(\mathrm{nm})\end{array}$ & $\begin{array}{c}\text { Particle } \\
\text { size }(\mathrm{nm}) \\
\pm\left(10^{-2}-10^{-1}\right)^{*}\end{array}$ \\
\hline & $\alpha-\mathrm{Fe}_{2} \mathrm{O}_{3}$ & 0.4951 & 0.5031 & 1.3734 & 59.37 \\
\hline $0 \mathrm{~h}$ & $\mathrm{CdO}$ & 0.2377 & 0.4691 & & 230.77 \\
\hline \multirow[t]{2}{*}{ CZF0h } & $\mathrm{ZnO}$ & 0.2672 & 0.3247 & 0.5200 & 193.59 \\
\hline & $\alpha-\mathrm{Fe}_{2} \mathrm{O}_{3}$ & 0.1953 & 0.5067 & 1.3638 & 37.31 \\
\hline $3 \mathrm{~h}$ & $\mathrm{CdO}$ & 0.1229 & 0.4638 & & 2.71 \\
\hline \multirow[t]{3}{*}{ CZF3h } & $\mathrm{ZnO}$ & 0.0082 & 0.3189 & 0.5375 & 50.00 \\
\hline & $(\mathrm{Zn}, \mathrm{Cd}) \mathrm{Fe}_{2} \mathrm{O}_{4}$ & 0.6734 & 0.8492 & & 3.99 \\
\hline & $\alpha-\mathrm{Fe}_{2} \mathrm{O}_{3}$ & 0.0114 & 0.5036 & 1.3749 & 50.00 \\
\hline $8 \mathrm{~h}$ & $\mathrm{CdO}$ & 0.0910 & 0.4670 & & 5.98 \\
\hline CZF8h & $(\mathrm{ZnCd}) \mathrm{Fe}_{2} \mathrm{O}_{4}$ & 0.8976 & 0.8494 & & 6.88 \\
\hline $20 \mathrm{~h}$ & $\mathrm{CdO}$ & 0.0393 & 0.4685 & & 4.95 \\
\hline CZF20h & $(\mathrm{Zn}, \mathrm{Cd}) \mathrm{Fe}_{2} \mathrm{O}_{4}$ & 0.9607 & 0.8494 & & 7.02 \\
\hline $25 \mathrm{~h}$ & $\mathrm{CdO}$ & 0.0361 & 0.4684 & & 7.17 \\
\hline CZF25h & $(\mathrm{Zn}, \mathrm{Cd}) \mathrm{Fe}_{2} \mathrm{O}_{4}$ & 0.9639 & 0.8490 & & 6.29 \\
\hline
\end{tabular}

${ }^{*}$ Error limits.

ratio with increasing magnetic field strength which may be explained by wave function shrinkage model [36]. The orbital magnetoconductivity theory predicts the forward interference among the random paths in the hopping process between the two sites spaced at a distance equal to optimum hopping distance resulting in positive magnetoconduction and can be expressed as

$$
\frac{\sigma(B, T)}{\sigma(0, T)} \approx 1+C_{\text {sat }} \frac{\frac{B}{B_{\text {sat }}(T)}}{1+\frac{B}{B_{\text {sat }}(T)}}
$$

where $C_{\text {sat }}$ is a temperature independent parameter and $B_{\text {sat }}$ is the magnetic field where the magnetoconductivity is saturated $\left[=0.7(h / e)(8 / 3)^{3 / 2}\left(1 / L_{l o c}^{2}\right)\left(T / T_{\text {mott }}\right)^{3 / 8}\right]$. Where $L_{l o c}$ is the localization length and $T_{\text {mott }}$ is the Mott characteristic temperature. In wave function shrinkage model, the average hopping length reduces due to the contraction of wave function of electrons under the influence of a magnetic field. As a result, the conductivity decreases with increasing magnetic field. Under a small magnetic field, the magnetoconductivity ratio can be expressed as [36]

$$
\ln \left[\frac{\sigma(B, T)}{\sigma(0, T)}\right]=-t_{1} \frac{e^{2} L_{l o c}^{4}}{\hbar^{2}}\left(\frac{T_{M o t t}}{T}\right)^{3 / 4} B^{2}
$$

where $t_{1}=5 / 2016$. The variation of magnetoconductivity ratio with magnetic field intensity is shown in Figure 7. The different points in Figure 7 are the experimental data for different samples and the solid lines are the theoretical best fit obtained from Equation (1) for milled samples and from Equation (2) for unmilled sample. For milled samples the value of $C_{s a t}$ and $B_{s a t}$ can be obtained as a fitting parameter of Equation (1) and the values are

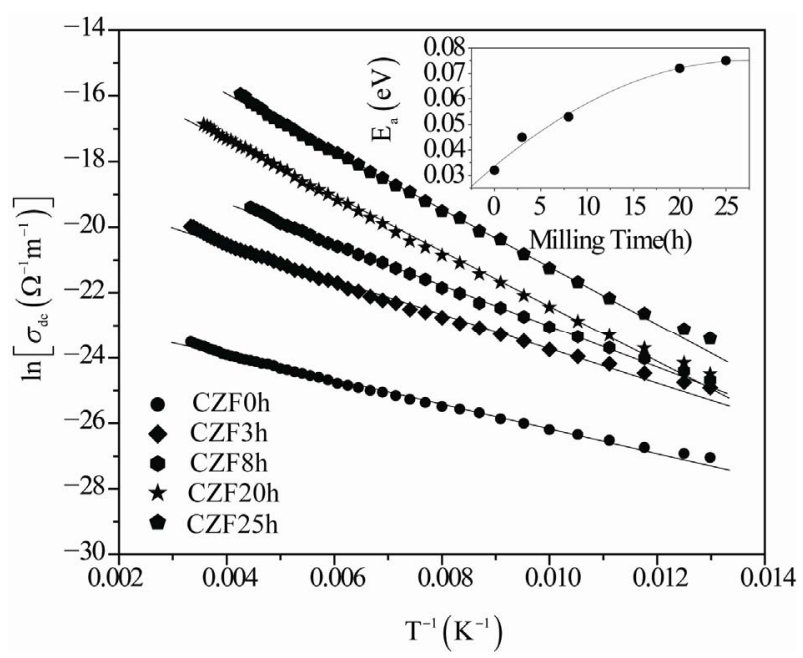

Figure 6. Variation of dc conductivity of the different samples with temperature. Inset shows the variation of activation energy of different samples with milling time. 




Figure 7. Variation of dc magnetoconductivity of different samples with magnetic field strength at $T=300 \mathrm{~K}$. The solid lines of milled samples are fitted with Equation (1) and the solid line of unmilled sample is fitted with Equation (2).

0.045 to 0.104 for $C_{\text {sat }}$ and 0.311 to $0.731 \mathrm{~T}$ for $B_{\text {sat, }}$ respectively. The values of $L_{l o c}$ of different investigated samples have been calculated. For the milled samples, the values of $L_{l o c}$ are $71.44 \mathrm{~nm}$ to $100.19 \mathrm{~nm}$ and for the unmilled sample, the localization length has been calculated as $26.79 \mathrm{~nm}$. Due to large localization length in milled samples conductivity is greater, which is confirmed by the dc conductivity result.

The ac conductivity of Cd-Zn ferrite samples are investigated in the frequency range $20 \mathrm{~Hz}$ to $1 \mathrm{MHz}$ and in the temperature range $77 \mathrm{~K} \leq T \leq 300 \mathrm{~K}$. A general feature of the amorphous semiconductors or disordered systems is that, in addition to the de conductivity contribution $\sigma_{\mathrm{dc}}$, the real part of complex ac conductivity $\sigma(f)$ is found to follow the so called universal dielectric response behavior, which can be expressed as [37-39]

$$
\sigma^{\prime}(f)=\sigma_{d c}+\sigma_{a c}(f)=\sigma_{d c}+\alpha f^{s}
$$

where $\sigma_{d c}$ is the dc conductivity, $\alpha$ is the temperature dependent constant and the frequency exponent $s \leq 1$. The value of $\sigma_{a c}(f)$ (the frequency dependent of conductivity) has been determined upon subtraction of the dc contribution from the total frequency dependent conductivity $\sigma^{\prime}(f)$. Figure 8 shows the linear variation of $\ln \left[\sigma_{a c}(f)\right]$ with $\ln (f)$ at different temperatures for the sample $25 \mathrm{~h}$. All the other samples behave in a similar manner. The value of ' $s$ ' has been calculated from the slope of these linear variation. Figure 9 shows the variation of ' $s$ ' with temperature. A weak variation of ' $s$ ' with temperature is observed up to $200 \mathrm{~K}$ but at higher temperature $(T>200 \mathrm{~K})$, the value of ' $s$ ' decrease with increasing temperature. In general, the conduction process of disordered

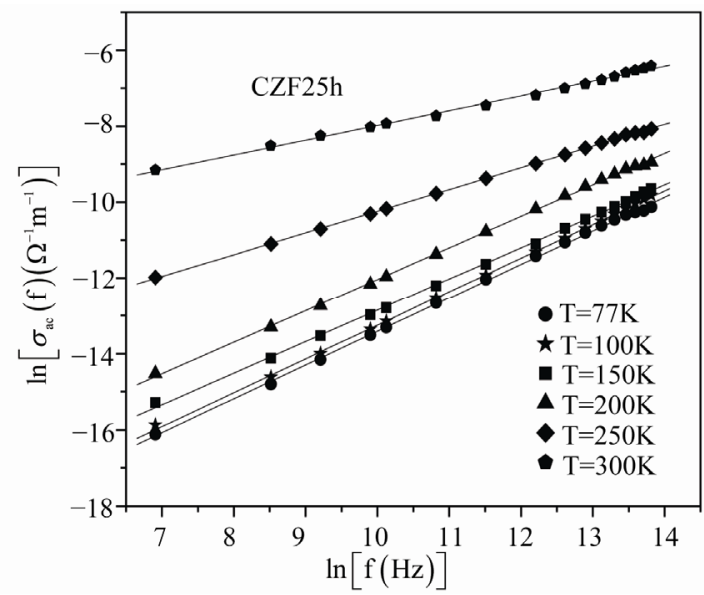

Figure 8. Variation of ac conductivity of CZF25h sample with frequency at different constant temperature.

systems is governed by two physical processes such as correlated barrier hopping $(\mathrm{CBH})$ [39] and quantum mechanical tunneling (electron tunneling [40], small polaron tunneling [39] and large polaron tunneling [38]. For different conduction process, the nature of temperature dependency of ' $s$ ' are different. So the exact nature of charge transport may be obtained experimentally from the temperature dependence of ' $s$ '. According to the electron tunneling theory ' $\mathrm{s}$ ' is independent of temperature, whereas for small polaron tunneling ' $s$ ' increases with increasing accordance with the CBH model. According to this model, the charge carrier hops between the sites over the potential barrier separating them and the frequency exponent ' $s$ ' can be written as temperature. But in case of correlated barrier hopping model ' $s$ ' only de-

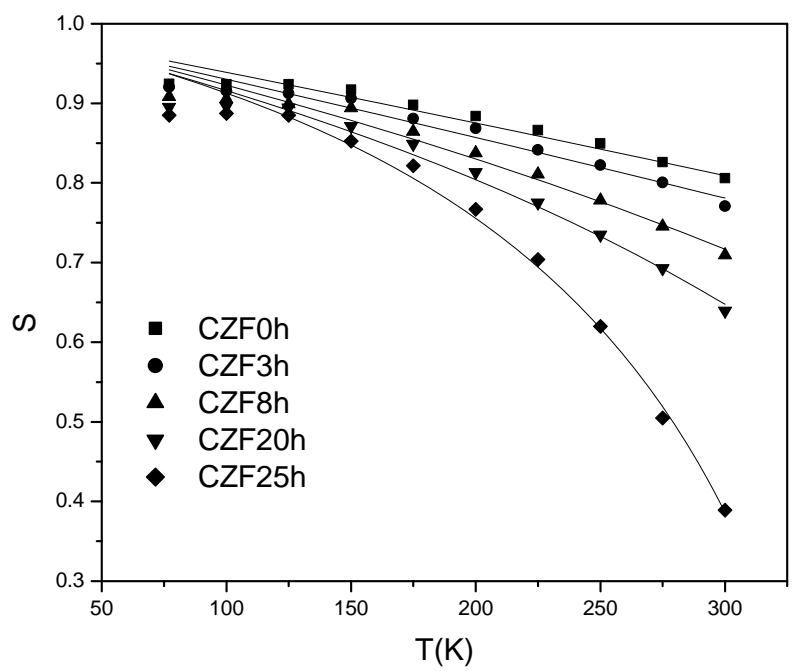

Figure 9. Variation of frequency exponent ' $s$ ' with temperature for different samples. The different solid lines are fitted with Equation (4). 
creases with the increasing temperature. The trend of variation of ' $s$ ' for the investigated samples show that it is in [39]

$$
s=1-\frac{6 k_{B} T}{W_{H}-k_{B} T \ln \left(\frac{1}{\omega \tau_{0}}\right)}
$$

where $W_{H}$ is the effective barrier height and $\tau_{0}$ is the characteristic relaxation time. For large value of $W_{H} / k_{B} T$, the value of ' $s$ ' can be considered as independent of frequency as there is a very small variation of ' $s$ ' with frequency [41]. Again for the investigated samples, the linear variation of $\ln \left[\sigma_{\mathrm{ac}}(f)\right]$ with $\ln (f)$ indicates that ' $s$ ' is frequency independent. Therefore the experimental data has been fitted with Equation (4) with $W_{H}$ and $\omega \tau_{0}$ as the fitting parameters. In Figure 9, the points are the experimental data and solid lines are the theoretical best fit values obtained from Equation (4). The best fitted values of the parameters $W_{H}$ and $\omega \tau_{\mathrm{o}}$ (at a fixed frequency of $f=$ $1 \mathrm{MHz}$ ) are in the range 0.82 to $1.34 \mathrm{eV}$ and $1.36 \times 10^{-14}$ to $7.63 \times 10^{-13} \mathrm{~s}$, respectively for different samples. $W_{H}$ has a greater value than the activation energy measured from grain and grain boundary contribution and the values of characteristic relaxation time $\tau_{0}$ has a similar value as expected for typical inverse phonon frequency. Therefore the trend of variation ' $s$ ' with temperature indicates that the ac conductivity of the investigated samples can be explained by the $\mathrm{CBH}$ model.

The temperature dependence of ac conductivity is shown in Figure 10 for the sample CZF25h for different some frequencies. A weak variation is observed at lower temperature $(T<200 \mathrm{~K})$ in comparison to high temperature $(T>200 \mathrm{~K})$. At a particular frequency the real part of complex conductivity increases with temperature and is found to follow a power law $\sigma^{\prime}(f) \propto T^{\mathrm{n}}$, which is shown as the solid lines in Figure 10. The values of $n$ have been calculated from the power law fitting and found to be strongly frequency dependent for all samples. For different frequency ranging from $1 \mathrm{kHz}$ to $1 \mathrm{MHz}$, the values of ' $n$ ' vary between 15.4 to 10.6 for CZF25h. According to the $\mathrm{CBH}$ model [37-40] the ac conductivity $\sigma^{\prime}(f)$ is expressed as $\sigma^{\prime}(f) \propto T^{2} R_{\omega}{ }^{6} \cong T^{\mathrm{n}}$ with $\mathrm{n}=[2+(1-$ s) $\left.\ln \left(1 / \omega \tau_{0}\right)\right]$ for broad band limit and $\sigma^{\prime}(f) \propto R_{\omega}{ }^{6} \cong T^{\mathrm{n}}$ with $\mathrm{n}=(1-\mathrm{s}) \ln \left(1 / \omega \tau_{0}\right)$ for narrow band limit, where $\mathrm{R}_{\omega}$ $=\mathrm{e}^{2} /\left\{\pi \varepsilon \varepsilon_{0}\left[W_{H}-k_{B} T \ln \left(1 / \omega \tau_{0}\right)\right]\right\}$. The theoretical values of ' $\mathrm{n}$ ', have been calculated taking $s=0.39$ at $300 \mathrm{~K}$ and 0.88 at $77 \mathrm{~K}$ for different frequencies and the value of $\tau_{0}$ $=3.26 \times 10^{-14} \mathrm{~s}$. The variation of the calculated values of ' $\mathrm{n}$ ' are in the range 15.61 to 11.40 at $300 \mathrm{~K}$ and 4.68 to 3.84 at $77 \mathrm{~K}$ for broad band limit and 13.61 to 9.40 at $300 \mathrm{~K}$ and 2.68 to 1.84 at $77 \mathrm{~K}$ for narrow band limit with frequency variation from $1 \mathrm{kHz}$ to $1 \mathrm{MHz}$. The ex-

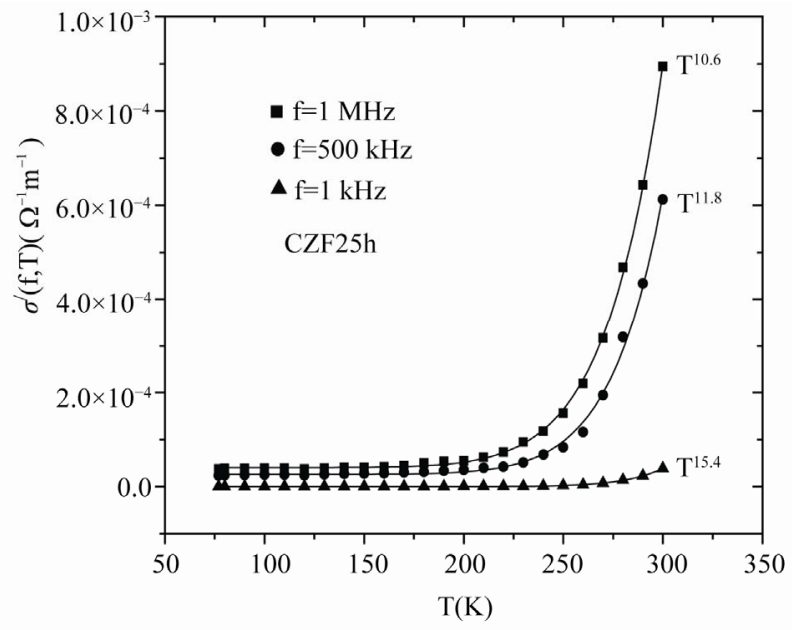

Figure 10. Variation of ac conductivity of CZF25h sample with temperature at different constant frequency. The lines are fitted with the equation $\sigma(f) \propto T^{\mathrm{n}}$.

perimental values are close to the theoretical values of broad band limit at the higher temperature range but at lower temperature, there is a discrepancy between theoretical and experimental result.

In general, interfacial polarization is exhibited by the ferrites due to structural inhomogenities and existence of free charges [42]. The hopping electrons at low frequencies may be trapped by the inhomogeneties. At a particular frequency, the increase in $\varepsilon^{\prime}(f)$ with temperature is due to the drop in the resistance of the ferrite with increasing temperature. Electron hopping is promoted by the low resistance and hence resulting a larger polarizability or larger $\varepsilon^{\prime}(f)$. The variation of real part of dielectric permittivity $\varepsilon^{\prime}(f)$ with frequency for different samples are shown in Figure 11 at $T=300 \mathrm{~K}$. The dielectric permittivity increases sharply with decreasing frequency for all the samples and such behaviour can be attributed to the presence of large degree of dispersion due to charge transfer within the interfacial diffusion layer present between the electrodes. The magnitude of dielectric dispersion depends on the temperature. At lower temperature the relaxation process becomes easier due to the freezing of the electric dipoles and thus there is decay in polarization with respect to the applied electric field. So a sharp increase in $\varepsilon^{\prime}(f)$ is observed at lower frequency region. Therefore the inhomogeneous nature of the samples containing different permittivity and conducting regions, governs the frequency behaviour of $\varepsilon^{\prime}(f)$ were the charge carriers are blocked by the poorly conducting regions.

The effective dielectric permittivity of this type of inhomogeneous systems is explained in terms of Maxwell Wegner capacitor model $[43,44]$ as which the complex impedance can be modelled by an equivalent circuit con- 


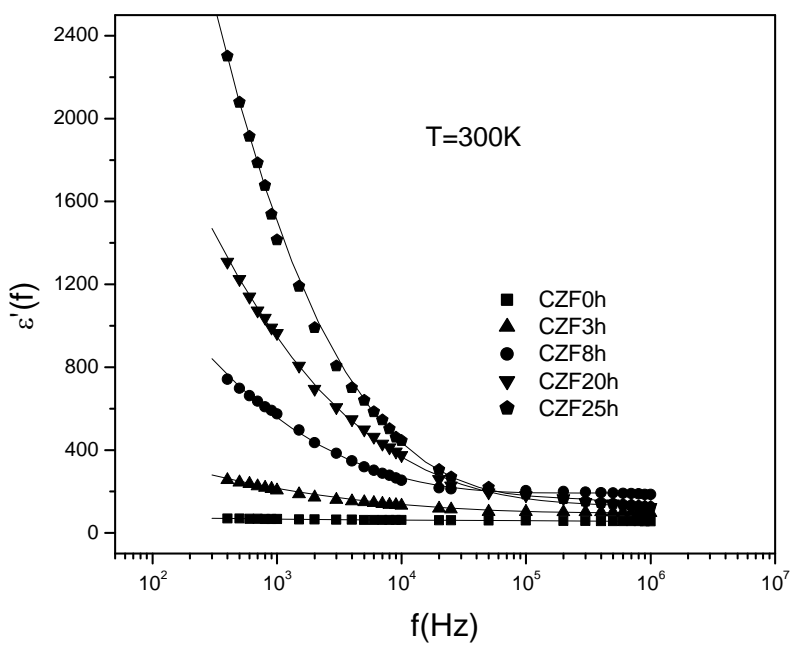

Figure 11. Variation of real part of dielectric permittivity of different samples with frequency at $\mathrm{T}=300 \mathrm{~K}$.

sisting of resistance and capacitance due to grain and interfacial grain boundary contribution and can be expressed as

$$
\begin{gathered}
Z=\frac{1}{i \omega C_{0} \varepsilon(\omega)}=Z^{\prime}-i Z^{\prime \prime} \\
Z^{\prime}=\frac{R_{g}}{1+\left(\omega R_{g} C_{g}\right)^{2}}+\frac{R_{g b}}{1+\left(\omega R_{g b} C_{g b}\right)^{2}}=\frac{\varepsilon^{\prime \prime}}{\omega C_{0}\left[\varepsilon^{\prime 2}+\varepsilon^{\prime \prime 2}\right]^{2}} \\
Z^{\prime \prime}=\frac{\omega R_{g}^{2} C_{g}}{1+\left(\omega R_{g} C_{g}\right)^{2}}+\frac{\omega R_{g b}^{2} C_{g b}}{1+\left(\omega R_{g b} C_{g b}\right)^{2}} \\
=\frac{\varepsilon^{\prime}}{\omega C_{0}\left[\varepsilon^{\prime 2}+\varepsilon^{\prime \prime 2}\right]^{2}}
\end{gathered}
$$

where sub-indexes ' $g$ ' and ' $g b$ ' refer to the grain and interfacial grain boundary respectively, $R$ is the resistance, $C$ is the capacitance, $\omega$ is $2 \pi f$ and $C_{0}$ is the free space capacitance. The real part of the complex impedance for different samples have been calculated from the experimental data for real $\left(\varepsilon^{\prime}\right)$ and imaginary $\left(\varepsilon^{\prime \prime}\right)$ part of the dielectric permittivity. Figure 12 shows the variation of the real part of the complex impedance of different samples with frequency at room temperature and Figure 13 shows the same variation for $20 \mathrm{~h}$ and $25 \mathrm{~h}$ samples in presence of a magnetic field. The points in both figures are the experimental data and the solid lines are the theoretical best fit obtained from Equation (6). It is observed from both figures that the experimental data are reasonably well fitted with the theory. The grain and grain boundary resistance and capacitance have been extracted from the analysis at room temperature whose

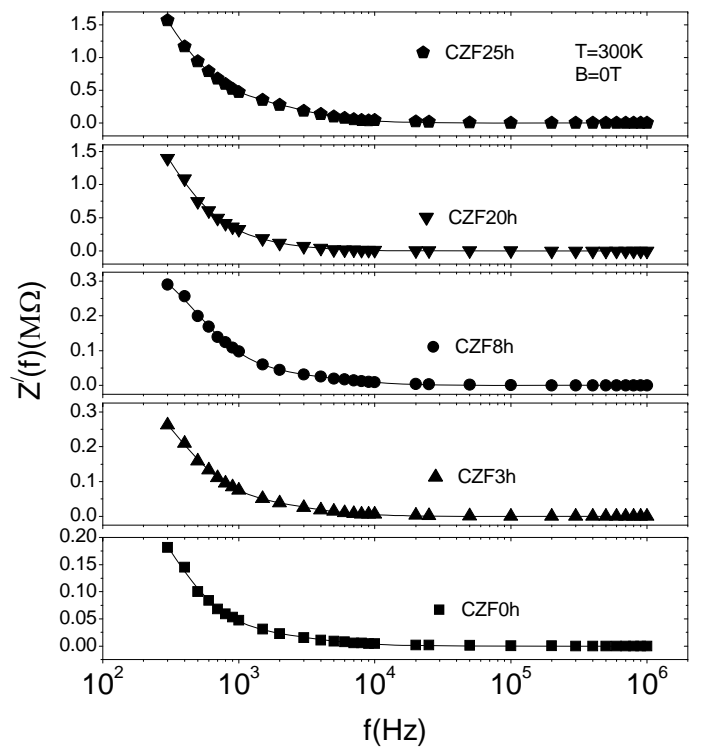

Figure 12. Variation of real part of impedance of different samples with frequency at room temperature. The solid lines are fitted with Equation (6).

value lie within the range $19.8 \mathrm{k} \Omega$ to $0.32 \mathrm{M} \Omega$ for $R_{g}$, $0.33 \mathrm{M} \Omega$ to $2.79 \mathrm{M} \Omega$ for $R_{g b}, 0.16$ to $1.94 \mathrm{nF}$ for $\mathrm{C}_{\mathrm{g}}$ and 0.20 to $1.62 \mathrm{nF}$ for $C_{g b}$ for different samples Q without magnetic field and $22.03 \mathrm{k} \Omega$ to $0.24 \mathrm{M} \Omega$ for $\mathrm{R}_{\mathrm{g}}, 0.35 \mathrm{M} \Omega$ to $2.05 \mathrm{M} \Omega$ for $R_{g b}, 0.18$ to $1.99 \mathrm{nF}$ for $C_{g}$ and 0.21 to $1.69 \mathrm{nF}$ for $C_{g b}$ for different samples in presence of a magnetic field. As the resistance due to interfacial grain boundary is much larger than the grain resistance, it may conclude that the grain boundary contribution dominates over the grain contribution.

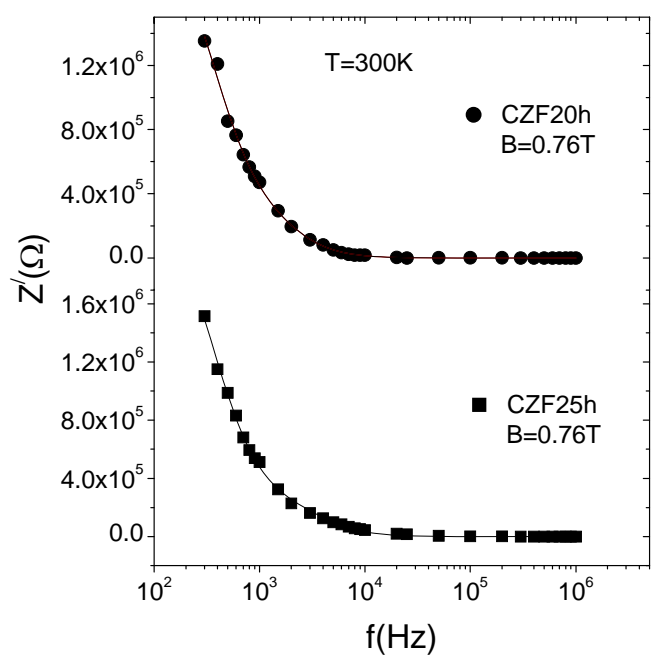

Figure 13. Variation of real part of impedance of CZF20h and CZF25h samples with frequency at room temperature in presence of magnetic field of $0.76 \mathrm{~T}$. The solid lines are fitted with Equation (6). 
The variation of real part of the ac conductivity for different samples at room temperature and $f=1 \mathrm{MHz}$ under the influence of magnetic field is shown in Figure 14. With increasing magnetic field, there is an increase in conductivity for different milled samples, but for unmilled sample opposite behaviour has been observed. At present, no theoretical model is found in literature which can explain directly the behaviour of ac conductivity in presence of magnetic field. The SEM micrograph reveals that the investigated samples are heterogeneous in nature with spherical grains. Thus the materials consist of grain and interfacial grain boundary regions. For such heterogeneous samples, it has been already discussed that the dielectric property and impedance depend on the grain and grain boundary resistance and capacitance. The real part of ac conductivity is related to the dielectric response by the relation $\sigma(B, f)=\omega \varepsilon_{0} \varepsilon^{\prime \prime}(B, f)$.As the value of $\varepsilon^{\prime \prime}(B, f)$ is dependent on the grain and grain boundary resistance and capacitances, the ac conductivity can be written as [45]

$$
\sigma^{\prime}(B, f)=\frac{d}{A\left(R_{g}+R_{g b}\right)} \frac{1-\omega^{2} \tau_{g} \tau_{g b}+\omega^{2} \tau\left(\tau_{g}+\tau_{g b}\right)}{1+\omega^{2} \tau^{2}}
$$

where $\tau_{g}=C_{g} R_{g}, \tau_{g b}=C_{g b} R_{g b}$ and $\tau=R_{g} R_{g b}\left(C_{g}+C_{g}\right) /\left(R_{g}\right.$ $\left.+R_{g b}\right)$. The change in the value of any of these resistance by the magnetic field will affect the value of the conductivity. From the analysis of the real part of complex impedance in presence of constant magnetic field at $0.76 \mathrm{~T}$, it has been found that the value of grain and grain boundary resistance increases by the application of magnetic field for unmilled sample, whereas it decreases for milled samples. Hence the total contribution due to grain and grain boundary resistance $\left(R=R_{g}+R_{g b}\right)$ decrease with increasing magnetic field for milled samples. Thus the influence on ac conductivity by magnetic field is due to the change in grain and grain boundary resistance by the applied magnetic field. But due to the inability of the analytical expression, the measured data can not be compared with the theory. Thus a more explicit theoretical and experimental study is required to reveal the true mechanism of magnetic field dependent ac conductivity.

\section{Conclusions}

The different Cd-Zn ferrite samples had been prepared by the high energy ball milling method. The samples were characterized by XRD which confirms the formation of normal spinel structure with tetrahedral vacancies with particle size $7 \mathrm{~nm}$. SEM picture reveals that the different samples consist of grains of almost spherical shape. The dc conductivity of different $\mathrm{Cd}-\mathrm{Zn}$-ferrite follows a simple hopping time of charge conduction mechanism. The magnetic field dependent conductivity of the differ-

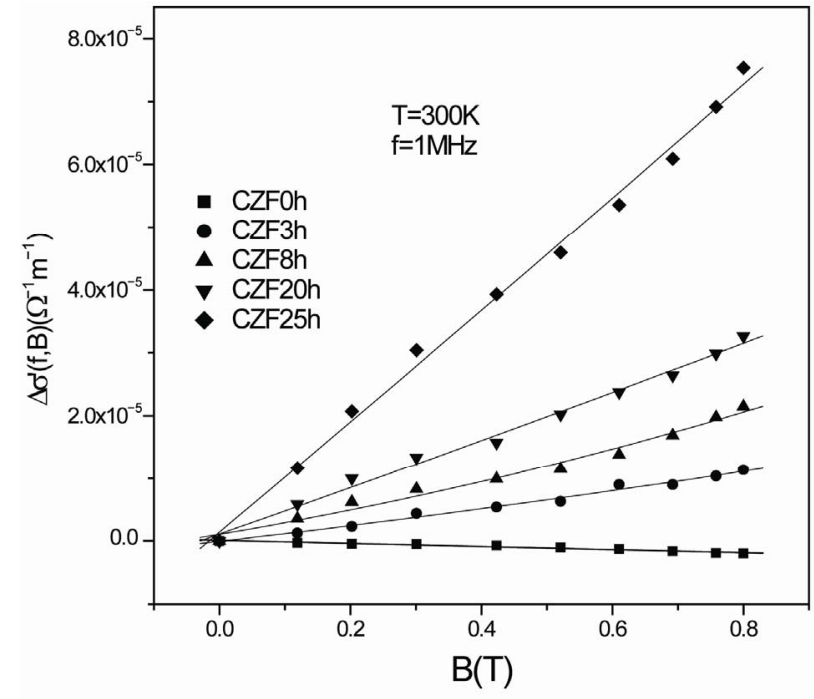

Figure 14. Variation of change in ac magnetoconductivity $\left[\Delta \sigma^{\prime}(f, B)=\sigma^{\prime}(f, B)-\sigma^{d}(f, 0)\right]$ with magnetic field intensity (B) at $T=300 \mathrm{~K}$ and frequency $f=1 \mathrm{MHz}$.

ent investigated samples increases with increasing magnetic field for milled samples whereas decreases with increasing magnetic field for unmilled sample and those can be explained in terms of orbital magneto conductivity theory and wave function shrinkage model respectively. The real part ac conductivity follows the power law $\sigma(f) \propto f^{\text {s }}$. The temperature dependence of universal dielectric response parameter 's' was found to follow correlated barrier hooping change transfer mechanism. At a particular frequency the conductivity of the investigated samples follows $\sigma^{\prime}(f) \propto \mathrm{T}^{\mathrm{n}}$, where the values of $\mathrm{n}$ strongly depend on frequency. The frequency dependent real part of complex permittivity shows large degree dispersion at low frequency, but rapid polarization at high frequencies which can be interpreted by Maxwell Wegner capacitor model. The grain resistance and capacitance was found to be smaller than the grain boundary resistance and capacitance and the total resistance due to grain and grain boundary decrease due to the application of a magnetic field. The ac resistivity of milled samples was found to show positive (increasing with increasing magnetic field) variation in presence of magnetic field which may be due to the variation of grain and grain boundary resistances by the application of a magnetic field.

\section{Acknowledgements}

This work has been carried out under Grant nos. F.27-1/2002.TS.V dated 19.03.2002 and F.28-1/2003. TS.V dated 31-03.2003 sanctioned by the MHRD, Government of India. The authors gratefully acknowledge the principal assistance received from the above organization 
during this work.

\section{REFERENCES}

[1] B. M. Berskovsky, V. F. Medvedev and M. S. Krakov, "Magnetic Fluids: Engineering Applications," Oxford University Press, Oxford, 1993.

[2] R. E. Ayala and D. W. Marsh, "Characterization and Long-Range Reactivity of Zinc Ferrite in High-Temperature Desulfurization Processes," Industrial \& Engineering. Chemistry Research, Vol. 30, No. 1, January 1991, pp. 55-60. doi:10.1021/ie00049a009

[3] L. A. Bissett and L. D. Strickland, "Analysis of a FixedBed Gasfier IGCC Configuration," Industrial \& Engineering. Chemistry Research, Vol. 30, No. 1, January 1991, pp. 170-176.

[4] M. Sugimoto, "The Past, Present and Future of Ferrites," Journal of the American Ceramic Society, Vol. 82, No. 2, February 1999, pp. 269-280. doi:10.1111/j.1551-2916.1999.tb20058.x

[5] I. Safarik and M. Sararikova, "Nanostructured Materials, Magnetic Nanoparticles and Biosciences," Springer, Wien, 2002, p. 1.

[6] S. Son, M. Taheri, E. Carpenter, V. G. Harris and M. E. Mctterny, "Synthesis of Ferrite and Nickel Ferrite NanoParticles Using Radio-Frequency Thermal Plasma Torch," Journal of Applied Physics,Vol. 91, No. 10, 2002, pp. 7589-7591. doi:10.1063/1.1452705

[7] I. Anton, I. de Sabata and L. Vekas, "Application Oriented Researches on Magnetic Fluids," Journal of Magnetism and Magnetic Materials, Vol. 85, No. 1-3, April 1990, pp. 219-226. doi:10.1016/0304-8853(90)90056-V

[8] R. D. McMickael, R. D. Shull, L. J. Swartzendruber, L. H. Bennett and R. E. Watson, "Magnetocaloric Effect in Superparamagnets," Journal of Magnetism and Magnetic Materials," Vol. 111, No. 1-2, June 1992, pp. 29-33. doi:10.1016/0304-8853(92)91049-Y

[9] D. L. Leslie-Pelecky and R. D. Rieke, "Magnetic Properties of Nanostructured Materials," Chemistry of Materials, Vol. 8, No. 8, August 1996, pp. 1770-1783. doi: $10.1021 / \mathrm{cm} 960077 \mathrm{f}$

[10] R. H. Kodama, A. E. Berkowitz, E. J. McNiff and S. Foner, "Surface Spin Disorder in Ferrite Nanoparticles (Invited)," Journal of Applied Physics, Vol. 81, No. 8, 1997, pp. 5552-5557.

[11] S. A. Oliver, H. H. Handeh and J. C. Ho, "Localized Spin Canting in Partially Inverted $\mathrm{ZnFe}_{2} \mathrm{O}_{4}$ Fine Powders," Physical Review B, Vol. 60, 1999, pp. 3400-3405. doi:10.1103/PhysRevB.60.3400

[12] S. Bid and S. K. Pradhan, "Preparation of Zinc Ferrite by High-Energy Ball Milling and Microstructure Characterization by Rietveld's Analysis," Materials Chemistry and Physics, Vol. 82, No. 1, September 2003, pp. 27-37. doi:10.1016/S0254-0584(03)00169-X

[13] S. K. Pradhan, S. Bid, M. Gateshki and V. Petkov, "Microstructure Characterization and Cation Distribution of
Nanocrystalline Magnesium Ferrite Prepared by Ball Milling," Materials Chemistry and Physics, Vol. 93, No. 1, September 2005, pp. 224-230. doi:10.1016/j.matchemphys.2005.03.017

[14] H. H. Hamdeh, J. C. Ho, S. A. Oliver, R. J. Willey, G. Oliveri and G. Busca, "Magnetic Properties of Partially-Inverted Zinc Ferrite Aerogel Powders," Journal of Applied Physics, Vol. 81, No. 4, 1997, pp. 1851-1857.

[15] P. Chen, D. Y. Xing, Y. W. Du, J. M. Zhu and D. Feng, "Giant Room-Temperature Magnetoresistance in Polycrystalline $\mathrm{Zn}_{0.41} \mathrm{Fe}_{2.59} \mathrm{O}_{4}$ with $\alpha-\mathrm{Fe}_{2} \mathrm{O}_{3}$ Grain Boundaries," Physical Review Letters, Vol. 87, No. 10, 2001, pp. 107202 (4).

[16] M. Hofmann, S. J. Campbell, H. Ehrhardt and R. Feyerherm, "The Magnetic Behaviour of Nanostructured Zinc Ferrites," Journal of Material Science, Vol. 39, No. 16-17, 2004, pp. 5057-5065. doi:10.1023/B:JMSC.0000039185.80910.59

[17] S. D. Shenoy, P. A. Joy and M. R. Anantharaman, "Effect of Mechanical Milling on the Structural, Magnetic and Dielectric Properties of Coprecipitated Ultrafine Zinc Ferrite," Journal of Magnetism and Magnetic Materials, Vol. 269, No. 2, February 2004, pp. 217-226. doi:10.1016/S0304-8853(03)00596-1

[18] Z. L. Lu, L. Y. Lv, J. M. Zhu, S. D. Li, X. C. Liu, W. Q. Zou, F. M. Zhang and Y. W. Du, "Magnetic and Transport Property Studies of Nanocrystalline $\mathrm{Zn}_{\mathrm{x}} \mathrm{Fe}_{3-\mathrm{x}} \mathrm{O}_{4}$," Solid State Communications, Vol. 137, No. 10, March 2006, pp. 528-532. doi:10.1016/j.ssc.2006.01.014

[19] Y. F. Tian, S. S. Yan, Y. P. Zhang, H. Q. Song, G. Ji, G. L. Liu, Y. X. Chen, L. M. Mei, J. P. Liu, B. Altuncevahir and V. Chakka, "Transformation of Electrical Transport from Variable Range Hopping to Hard Gap Resistance in $\mathrm{Zn}_{\mathrm{x}} \mathrm{Fe}_{3-\mathrm{x}} \mathrm{O}_{1-\mathrm{v}}$," Journal of Applied Physics, Vol. 100, No. 10, 2006, pp. 103901 (6).

[20] L. D. Tung, V. Kolesnichenko, G. Caruntu, D. Caruntu, Y. Remond, V. O. Golub, C. J. O’Connor and L. Spinu, “Annealing Effects on the Magnetic Properties of Nanocrystalline Zinc Ferrite," Physica B, Vol. 319, No. 1-4, July 2002, pp. 116-121. doi:10.1016/S0921-4526(02)01114-6

[21] P. M. G. Nambissan, C. Upadhyay and H. C. Verma, "Position, Lifetime Spectroscopic Studies of Nanocrystalline $\mathrm{ZnFe}_{2} \mathrm{O}_{4}$," Journal of Applied Physics, Vol. 93, No. 10, 2003, pp. 6320-6326. doi:10.1063/1.1569973

[22] C. N. Chinnasamy, A. Narayanasamy, N. Ponpandian, K. Chattopadhyay, H. Guerault and J. M. Greneche, "Magnetic Properties of Nanostructured Ferimagnetic Zinc Ferrite," Journal of Physics: Condensed Matter, Vol. 12, No. 35, September 2000, pp. 7795. doi:10.1088/0953-8984/12/35/314

[23] H. Ehrhardt, S. J. Capbell and M. Hofmann, "Structural Evolution of Ball Milled $\mathrm{ZnFe}_{2} \mathrm{O}_{4}$," Journal of Alloys and Compounds, Vol. 339, No. 1-2, June 2002, pp. 255-260. doi:10.1016/S0925-8388(01)02011-4

[24] D. Ravinder, "Thermoelectric Power and Electrical Resistivity of Cadmium-Substituted Manganese Ferrite," 
Materials Letters, Vol. 44, No. 3-4, June 2000, pp. 130138. doi:10.1016/S0167-577X(00)00015-X

[25] D. Ravinder, "Electrical Transport Properties of Cadmium Substituted Copper Ferrite," Materials Letters, Vol. 43, No. 3, April 2000, pp. 129-138. doi:10.1016/S0167-577X(99)00245-1

[26] D. Ravinder, S. S. Rao and P. Shalini, "Room Temperature Electric Properties of Cadmium-Substituted Nickel Ferrites," Materials Letters, Vol. 57, No. 24-25, August 2003, pp. 4040-4042. doi:10.1016/S0167-577X(03)00089-2

[27] H. M. Rietveld, "Line Profile of Neutron Powder Diffraction Peaks for Structure Refinement," Acta Crystallographica, Vol. 22, 1967, pp. 151-152. doi:10.1107/S0365110X67000234

[28] H. M. Rietveld, "A Profile Refinement Method for Nuclear and Magnetic Structures," Journal of Applied Crystallography, Vol. 2, 1969, pp. 65-71. doi:10.1107/S0021889869006558

[29] R. A. Young, "The Rietveld Method," Oxford University Press, Oxford, 1996.

[30] L. Lutterotti, P. Scardi and P. Maistrelli, "LSI-a Computer Program for Simultaneous Refinement of Material Structure and Microstructure," Journal of Applied Crystallography, Vol. 25, No. 3, 1992, pp. 459-462. doi:10.1107/S0021889892001122

[31] L. Lutterotti, "MAUD Version 2.046.," http://www.ing.unitn.it/ luttero/maud.

[32] S. Ghatak, M. Sinha, A. K. Meikap and S. K. Pradhan, "Electrical Transport Properties of Nanocrystalline Zinc Ferrite," Physica E, Vol. 40, January 2008, pp. 26862693. doi:10.1016/i.physe.2007.12.030

[33] C. A. Neugebauer and M. B. Webb, "Electrical Conduction Mechanism in Ultrathin Evaporated Metal Films," Journal of Applied. Physics, Vol. 33, No. 1, 1962, pp. 74-83.

[34] V. L. Nguyen, B. Z. Spivak and B. I. Shklovskii, "Tunnel Hops in Disordered Systems," Soviet Physics-JETP, Vol. 62,1985, p. 1021.
[35] U. Sivan, O. Entin-Wohiman and Y. Imry, "Orbital Magnetoconductance in the Variable Range Hopping Regime," Physical Review Letters, Vol. 60, No. 15, 1988, pp. 15661569. doi:10.1103/PhysRevLett.60.1566

[36] B. I. Shklovskii, "Positive Magnetoresistance in the Variable Range Hopping Conduction Regimes," Soviet Physics Semiconductors, Vol. 17, 1983, p. 1311.

[37] N. F. Mott and E. Davis, "Electronic Process in Noncrystalline Materials," 2nd Edition, Oxford University Press, New York, 1997.

[38] A. R. Long, "Frequency-Dependent Lossin Amorphous Semi-Conductors," Advances in Physics, Vol. 31, No. 5, 1982, pp. 553-637. doi:10.1080/00018738200101418

[39] S. R. Elliott," A.c. Conduction in Amorphous Chalcogenide and Pnictide Semiconductors," Advances in Physics, Vol. 36, No. 2, 1987, pp. 135-217. doi:10.1080/00018738700101971

[40] A. L. Efros, "On the Theory of A.c. Conduction in Amorphous Semiconductors and Chalcogenide Glasses," Philosophical Magazine B, Vol. 43, No. 5, 1981, pp. 829-838. doi:10.1080/01418638108222349

[41] B. G. Soares, M. E. Leyva, G. M. O. Barra and D. Khastgir, "Dielectric Behaviour of Polyaniline Synthesized by Different Techniques," European Polymer Journal, Vol. 42, No. 3, 2006, pp. 676-686. doi:10.1016/j.eurpolymj.2005.08.013

[42] S. S. Suryavanshi, S. R. Patil, S. A. Patil and S. R. Sawant, "D.C. Resistivity and Dielectric Behaviour of $\mathrm{Ti}^{4+}$ Substituted Mg-Zn Ferrite," Journal of the Less-Common Metals, Vol. 168, No. 2, March 1991, pp. 169-174. doi:10.1016/0022-5088(91)90298-I

[43] J. C. Maxwell, "A Treatise on Electricity and Magnetism," Oxford University Press, Oxford, 1988.

[44] V. Hippel, "Dielectrics and Waves," Wiley, New York, 1954.

[45] G. Catalan, "Magnetocapacitance without Magnetoelectric Coupling," Applied Physics Letters, Vol. 88, No. 10, 2006, pp. 102902-102905. doi:10.1063/1.2177543 